\title{
Traumatic Intracranial Frontal Extradural Hematoma Associated with Orbital Subperiosteal Hematoma.
}

\author{
M K Gupta, K Dhungel, P L Sah, K Ahmad, R K Rauniyar
}

Department of Radio diagnosis \& Imaging, BPKIHS, Dharan, Nepal

\begin{abstract}
Intracranial extradural hematoma $(\mathrm{EDH})$ is a frequent lesion but simultaneous occurrence of EDH with orbital subperiosteal hematoma following head injury is a rare event. We report a 22 year old male who sustained head injury during road traffic accident. Glasgow coma scale was $13 / 15$ on arrival and he had left orbital ecchymosis \& subconjunctival hemorrhage. CT revealed left frontal extradural hematoma with associated orbital subperiosteal hematoma. No bone fracture was seen. The patient had good clinical recovery following surgical evacuation of the hematomas.
\end{abstract}

Keywords: head injury, frontal extradural hematoma, orbit, subperiosteal hematoma.

\section{Introduction}

Intraorbital hematoma is an uncommon entity, usually associated with ophthalmologic procedures, coagulation disturbances, or trauma. Post traumatic orbital subperiosteal hematoma is rarely reported in the literature and simultaneous occurrence of intracranial extradural hematoma $(\mathrm{EDH})$ with orbital subperiostealhematoma is even rare. There can be associated orbital roof fracture. We report a rare case of post traumatic simultaneous occurrence of intracranial frontal EDH with orbital subperiosteal hematoma in a 22 year old male without associated bony fracture.

Correspondence to: Dr Mukesh Kumar Gupta, Assistant Professor, Department of Radiodiagnosis, BPKIHS, Dharan, Nepal Email: mukeshgupta148@yahoo.com

\section{Case Report}

A 22 year old male was brought to the casualty following road traffic accident. Glasgow coma scale was $13 / 15$ on arrival and he had left orbital ecchymosis and subconjunctival hemorrhage. Vision in both eyes was intact and fundus examination revealed no abnormality. Limited superior gaze was observed in left eye. CT scan of brain and orbit was performed which revealed a large intracranial frontal EDH on left side and associated ipsilateral subperiosteal hematoma in the superior wall of the orbit causing downward displacement of the globe (Fig $1 \& 2$ ). No fracture was seen. No continuity between these two hematomas was observed. The patient had a good clinical recovery following surgical evacuation of the hematoma.

\section{Discussion}

Orbital hematomas are classified by Landa ${ }^{1}$ as either intraorbital or subperiosteal. 
Subperiosteal hematoma can be spontaneous or traumatic in origin ${ }^{2}$. These hematomas develop between the bone and separated periosteum in the orbital roof. This usually occurs as a result of direct rupture of subperiosteal blood vessels or as an extension of a subgaleal hematoma ${ }^{3}$. The frontal bone forms the largest continuous concave bony orbital surface, and the orbital periosteum is not firmly adhered to the bone. The attachment is stronger along suture lines between the bones forming the orbit ${ }^{4}$. In our case also the hematoma was located at the roof of the orbit.

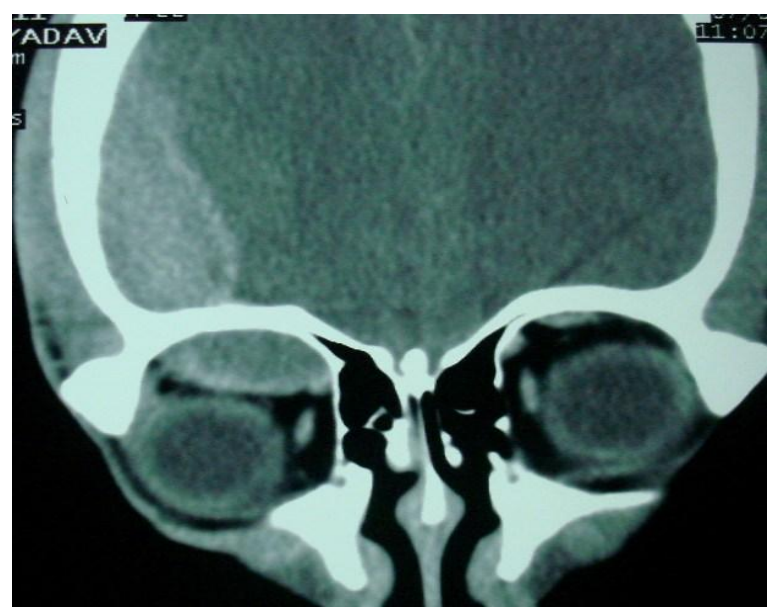

Figure 1: CT scan brain \& orbit (coronal section) showing left frontal EDH and orbital subperiosteal hematoma causing downward displacement of the globe.

Subperiosteal hematoma of the orbital roof occurs almost exclusively in children or young adults. They are less likey to occur later since the periosteal bony connection may grow firmer with age ${ }^{3}$. Age of the patient in our case was 22 years.

CT signs of subperiosteal hematoma include (a) sharply defined, high attenuation mass (blood density) with broad base abutting the superior orbital roof (b) inferior displacement of the orbital contents, and (c) optic nerve stretching. The differential diagnosis includes neoplasm and inflammation. However, when the clinical presentation is combined with the CT, a diagnosis should be easily established ${ }^{3}$.
Chronic hematic intraorbital cyst can result from undiagnosed or untreated intraorbital hematoma ${ }^{5}$.

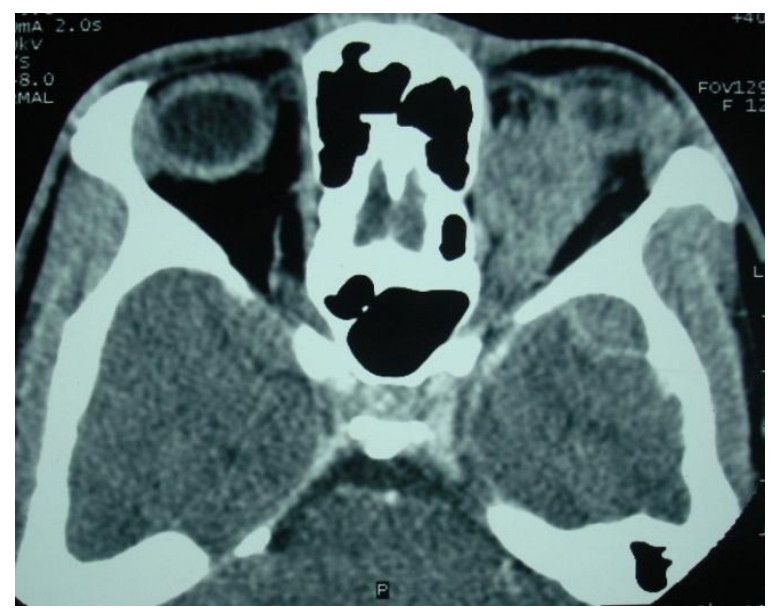

Figure 2: CT scan of orbit (axial section) showing subperiosteal hematoma in the superior part of left orbit.

\section{References}

1. Landa MS, Landa EH, Levine MR. Subperiosteal hematoma of the orbit: case presentation. Ophthal Plastic Reconst Surg 1988;3:189-192.

2. Nakai K, Doi E, Kuriyama T, et al. Spontaneous subperiosteal hematoma of the orbit. Surg Neurol 1983; 20:100-102.

3. Seigel RS, Williams AG, Hutchison JW, et al. Subperiosteal hematoma of the orbit: angiographic and computed tomographic diagnosis. Radiology 1982;143: 711-714.

4. Wolter JR. Subperiosteal hematoma of the orbit in young males: a serious complication of trauma or surgery in the eye region. Trans Am Ophthalmol Soc 1979;77:104-120.

5. Goldberg SH, Sassani JW,Parnes RE. Traumatic intraconal hematic cyst of the orbit. Arch Ophthalmol 1992;110:378-380. 